\section{La comunicazione web dei quartieri fieristici}

\author{
Tonino Pencarelli - Marco Cioppi - Giancarlo Ferrero \\ Ilaria Curina
}

\begin{abstract}
Obiettivo del paper: Obiettivo del lavoro è delineare le tendenze ed il ruolo assunto dalla comunicazione web all' interno dello scenario fieristico internazionale, approfondendo l'adeguatezza della comunicazione online degli Exhibition centers in Italia.

Metodologia: Il contributo adotta un processo di systematic review per identificare tutti gli articoli accademici sui Trade Shows (TSs) fra il 1996 ed il 2016 (evidenziando, nello specifico, il ruolo della comunicazione web) e un modello di valutazione della qualità dei siti web adattato con una serie di quality variables specificatamente identificate in letteratura.

Risultati: Dall'indagine emerge un impiego ancora deficitario ed arretrato della comunicazione online da parte dei poli fieristici nazionali. Nonostante la quasi totalità dei quartieri italiani possieda oggi un sito web, risultano essere ancora pochi i quartieri che adottano una comunicazione web realmente interattiva.

Limiti: Il limite dello studio si collega al fatto che l'indagine è stata realizzata da una prospettiva esterna, non rendendo possibile, in questo ambito, effettuare una valutazione della comunicazione web in relazione agli specifici obiettivi delle governance fieristiche.

Implicazioni pratiche: Lo studio individua una serie di possibili percorsi di innovazione che il comparto fieristico nazionale potrebbe intraprendere per ridurre il gap che attualmente lo separa da un uso totalmente performante degli strumenti comunicativi del web.

Originalità del lavoro: La review ha permesso di mappare gli articoli accademici che si sono occupati, negli ultimi 20 anni, di approfondire lo studio sui Trade Shows, consentendo di delineare le principali tendenze della recente letteratura di settore dedicata a tale filone di ricerca.
\end{abstract}

Parole chiave: comunicazione web; quartieri fieristici; trend fieristici; website evaluation; Trade Shows.

Purpose of the paper: The main goal is to outline the trends and role played by the web communication within the international Trade Fair scenario. Then, the adequacy of the online communicative activities, adopted by the Italian Exhibition centers, is evaluated.

Methodology: A systematic review (aimed at identify the academic articles focused on the Trade Shows field between 1996 and 2016 and in particular on the web communication role) and a website quality evaluation model are adopted. The model is here adapted by the insertion of a series of quality variables used in literature for the Trade Show websites quality evaluation. 
sinergie Vol. 35, N. 102, 2017

Results: The survey shows an overall underdeveloped adoption of the online communicative tools by the National Exhibition centers. Although the majority of them own a website, only a small percentage realizes really interactive online communicative activities.

Limits: The limitation is linked to the fact the survey has been conducted from an external perspective, not making possible to realize a Trade Show online communication assessment in relation to the governance objectives.

Implications: The study identifies a series of learning and innovative paths the National industry could undertake in order to reduce the gap that currently separates the National Exhibition universe from a totally powerful use of the web communication tools.

Originality of the paper: The review has permitted to map the different academic articles that have deepened, in the last 20 years, the Tradeshows field, allowing to outline the main trends characterizing the recent literature specifically dedicated to this sector.

Key words: web communication; exhibition centers; trade show trends; website evaluation; trade shows.

\section{Introduzione}

Definite nel 1986 come "eventi che riuniscono, in un unico luogo fisico, gruppi di fornitori e di distributori aventi come principale obiettivo quello di presentare i propri prodotti/servizi appartenenti ad un medesimo settore/ disciplina" (Black, 1986), le fiere rappresentano ancora oggi un business di primo piano ed un volano per l'economia mondiale ed europea (Tafesse, 2014). Con 465 centri fieristici, il continente europeo detiene più del $50 \%$ della superficie espositiva mondiale (AEFI, 2016), con Germania, Italia e Francia che dominano l'intero settore in termini di sedi, di espositori e di visitatori (UFI 2016; Li, 2015; Chu e Chiu, 2013; Smith et al., 2004).

In particolare, in un paese come l'Italia, il cui sistema industriale è composto principalmente da piccole e medie imprese, le manifestazioni fieristiche rappresentano ancora oggi uno degli strumenti più efficaci per promuovere prodotti e servizi, contattare nuovi clienti e soprattutto per ottenere l'ingresso in nuovi mercati (secondo dati AEFI 2016, per l'88,5\% delle PMI nazionali le fiere continuano a rappresentare un palcoscenico comunicativo di primaria importanza). Nel 2016, nei quartieri fieristici italiani sono state programmate 946 manifestazioni, 189 delle quali a carattere internazionale. Nel complesso, ogni anno l'industria fieristica movimenta 22 milioni di visitatori, genera un giro d'affari pari a 60 miliardi di euro ed un fatturato pari a 2 miliardi, con rilevanti ricadute per le città ospitanti, in termini di trasporti, ristorazione e di strutture alberghiere (AEFI, 2016; Tafesse, 2014).

Diventato argomento sistematico di ricerca nel corso del decennio 1980-1990 (Soilen, 2010), gli studi dedicati alle fiere (Trade Show) si sono principalmente concentrati, in quegli anni, sulla importanza di tale strumento in una ottica prettamente promozionale/di vendite (Aloui e Jebsi, 2016; Li, 2015; Rodriguez et al., 2015; Çobanoğlu e Turaeva, 2014; 
Soilen, 2010; Tafesse e Korneliussen, 2012; Kirchgeorg et al., 2010; Power e Jansson, 2008; Herbig et al., 1998).

In particolare, le fiere venivano percepite unicamente come luoghi di incontro domanda-offerta dove l'obiettivo principale, per l'espositore, era aggiudicarsi la vendita prima del termine della manifestazione stessa (Çobanoğlu e Turaeva 2014). Il focus quindi era principalmente indirizzato sui selling objectives dello strumento fiera a scapito dei non selling (Menon e Manoj, 2013).

Negli ultimi anni, si assiste ad una inversione di tendenza: le fiere non vengono più percepite unicamente come uno strumento di vendita e di promozione fine a se stesso, ma diventano parte vitale del marketing mix (Rinallo et al., 2016; Sasaka, 2012), una leva strategica di marketing in grado di conseguire obiettivi di vendita e non (Çobanoğlu e Turaeva, 2014; Soilen, 2010). Racchiudendo in sé le potenzialità della comunicazione di massa, tipica della pubblicità, delle strategie di profilazione, connotative della direct mail, della vendita diretta e delle strategie di networking, caratteristiche dei nuovi media, le fiere acquisiscono nuove funzioni e nuovi ruoli (Li, 2015; Chu e Chiu, 2013; Sasaka, 2012).

Accanto quindi agli obiettivi di vendita la letteratura di settore degli ultimi anni identifica nuove funzioni che le fiere potrebbero esercitare in qualità di piattaforme relazionali, dove i diversi attori partecipanti (espositori, visitatori, organizzatori, poli fieristici, città, ecc.) rispondono non solo a stimoli commerciali (Andreae et al., 2013), ma anche e soprattutto di information/knowledge exchange (Li, 2015).

Il focus si sposta dai selling objectives ai non selling, in grado anchessi, in maniera differente, di influenzare, nel lungo termine, le future decisioni di acquisto dei visitatori fieristici (Chu e Chiu, 2013). Le fiere diventano così uno strumento non solo promozionale/di vendita, ma soprattutto di scambio relazionale/informativo in grado di contribuire alla 1) costruzione della brand identity delle aziende partecipanti (Chu e Chiu, 2013); 2) instaurazione di rapporti informativi e relazionali fra gli stakeholder fieristici (Li, 2015); 3) presentazione/trasferimento degli sviluppi tecnologici/innovativi dei prodotti e dei servizi presenti negli stand (Aloui e Jebsi, 2016); 4) trasferimento informativo, cooperazione e future relazioni commerciali (Cop, Kara, 2014); 5) learning/knowledge creation; accesso a nuove tecnologie, trend di mercato e potenziali partner (Rodriguez et al., 2015); 6) customer relationship building, competitive intelligence e market information gathering (De Freitas e Da Silva, 2013; Tafesse e Korneliussen, 2012); 7) costruzione di network relazionali (Soilen, 2010; Evers e Knight, 2008); 8) strategie di benchmarking (Çobanoğlu e Turaeva, 2014).

Le fiere, quindi, rappresentano da anni un tema di grande interesse e al contempo in forte mutamento (Gottlieb et al., 2014; Tafesse, 2014; Prado-Roman et al., 2012; Tafesse e Korneliussen, 2011). Un mutamento riconducibile ad una evoluzione dei ruoli, delle funzioni e della natura stessa della fiera (Rodriguez et al., 2015), in seguito anche ai recenti cambiamenti socio-economici che hanno caratterizzato l'ultimo decennio (Rodriguez et al., 2015; Menon e Manoi, 2013; Kirchgeorg et al., 2010). Di fronte a questo scenario e sulla base di queste preliminari considerazioni, il presente contributo si prefigge un duplice obiettivo: a) identificare le principali
Tonino Pencarelli

Marco Cioppi

Giancarlo Ferrero

Ilaria Curin

La comunicazione web dei quartieri fieristici 
sinergie Vol. 35, N. 102, 2017

tendenze emergenti dalla recente letteratura internazionale dedicata al filone Trade Shows (TSs); b) capire il ruolo assunto dalla comunicazione web all'interno di tale scenario fieristico, valutando, l'adeguatezza delle attività comunicative online attualmente adottate in Italia da uno dei principali stakeholder fieristici di interesse (Gopalakrishna et al., 2010): gli Exhibition centers. Nello specifico il lavoro cercherà di rispondere alle seguenti domande di ricerca:

$R Q$ (1) Quali sono le principali tendenze emergenti dalla recente letteratura di settore dedicata al filone Trade Shows (TSs)?

$R Q$ (2) Alla luce delle tendenze in atto, che ruolo assume la comunicazione web all interno dell'attuale scenario fieristico internazionale?

$R Q$ (3) Alla luce del ruolo assunto dalla comunicazione web in ambito fieristico, i poli fieristici nazionali adottano attività di comunicazione web adeguate?

Per rispondere a questi interrogativi, dopo aver delineato le principali tendenze, mediante una review della letteratura degli ultimi 20 anni (19962016), si procederà con una analisi dei siti web ufficiali di tutti i quartieri nazionali attualmente attivi, avvalendosi di un modello (2QCV2Q; Mich e Franch, 2000) specificatamente adattato per la valutazione della qualità web fieristica. I dettagli della metodologia sono esposti nel $\$ 2$ e 3 .

\section{Background teorico e review della letteratura}

Al fine di delineare come le principali tendenze caratterizzanti la letteratura di settore dedicata al filone Trade Show (TSs) siano evolute nel corso degli ultimi 20 anni, si è proceduto con una literature review articolata nelle seguenti fasi: raccolta, sistematizzazione/selezione ed analisi approfondita del campione di pubblicazioni identificato. Nella fase di raccolta è stata condotta una ricerca sistematica (Ricerca avanzata con le key word "Trade Show", "Trade Fair", "Exhibition" e "Fair" nel titolo del contributo; Intervallo temporale: 1996-2016), mediante la consultazione dei seguenti Database: 1. Google Scholar; 2. Scopus; 3. Elsevier ScienceDirect; 4. Repec (IDEAS).

Nella fase di sistematizzazione/selezione, partendo dai contributi iniziali identificati (1.899), ripartiti fra i diversi motori di ricerca, sono stati presi in considerazione tutti gli articoli accademici (459). In un secondo momento, si è proceduto con la lettura degli abstract dei 459 lavori al fine di eliminare gli articoli non in linea con l'obiettivo della ricerca. Al termine di questa fase, si è giunti ad un campione finale composto da 188 pubblicazioni che si sono specificatamente occupate dello studio dei Trade Shows (TSs) fra il 1996 ed il 2016. In questa fase è stato, inoltre, possibile risalire ai Journal che hanno pubblicato il maggior numero di articoli sul tema: Journal of Business and Industrial Marketing (15), Industrial Marketing Management (10), Journal of Convention and Event Tourism (5), Journal of Promotion Management (5). Dal punto di vista temporale (Fig. 1), emerge come l'interesse della letteratura di settore per la tematica sia 
considerevolmente aumentato nel corso degli ultimi anni (in particolare nel corso del 2008), raggiungendo un picco massimo nel 2015, seguito da una successiva fase di calo (2016).

Fig. 1: Articoli per anni

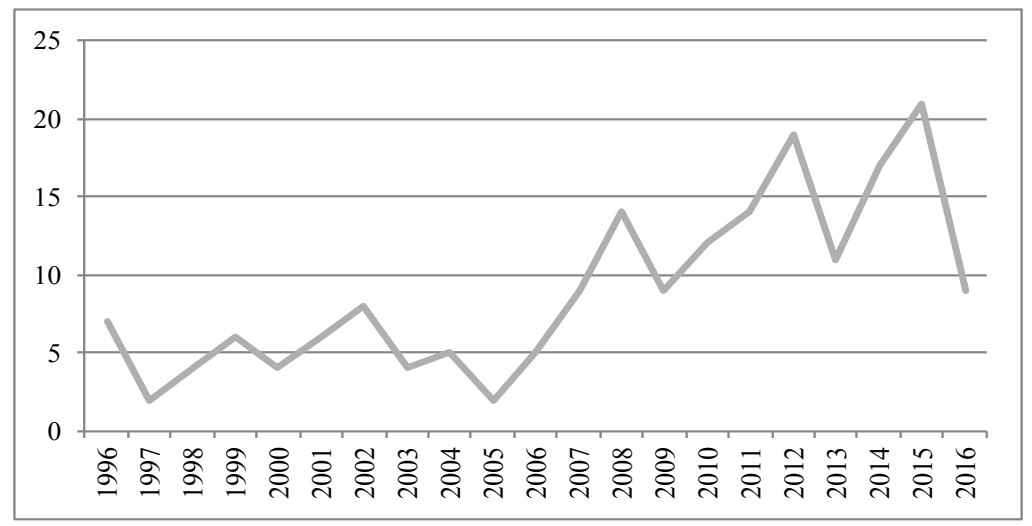

Fonte: Nostra elaborazione

In un' ottica metodologica, più della metà degli articoli (55\%) è di natura empirico-quantitativa (survey, questionari, modelli statistici), mentre la restante percentuale (45\%) adotta metodologie di carattere qualitativo (Review focalizzate su specifiche tematiche afferenti al filone Trade Show, Case studies, interviste in profondità). Anche se alcuni lavori empirici si focalizzano sulla comparazione fra Trade Shows appartenenti a sistemi fieristici geografici differenti, nella maggior parte dei casi i lavori non analizzano settori fieristici specifici, ma si occupano dello strumento fiera in una ottica manageriale. Dal punto di vista geografico, infine, emerge come la letteratura si sia interessata ad approfondire l'importanza dello strumento fiera, sia nei paesi sviluppati, che in quelli emergenti con una prevalenza di studi condotti in Europa (34\%), in Asia (33\%) ed in America (24\%).

Dal punto di vista dei temi affrontati, dalla lettura dei full text dei 188 articoli accademici del campione, emerge come alcuni argomenti di ricerca abbiano caratterizzato l'intero periodo di riferimento (1996-2016), confermandosi come aree tematiche di grande interesse degli ultimi 20 anni.

In particolare, emerge come la letteratura si sia costantemente occupata di approfondire l'importanza dell'evento fiera per le imprese. Evento considerato strumento vitale del marketing mix (Cop e Kara, 2014; Menon e Manoj, 2013; Ahola, 2012; Kirchgeorg et al., 2010; Blythe, 2009; LingYee, 2006; Blythe, 2002; Palumbo e Herbig, 2002; Munuera e Ruiz, 1999; Herbig et al., 1997) in grado di contribuire alla creazione di rapporti fra i diversi stakeholder fieristici (Remolar et al., 2015; Tafesse e Skallerud, 2015; Sarmento et al., 2014; Rinallo e Golfetto, 2011; Kirchgeorg et al., 2009; LingYee, 2007; Blythe, 2002; Hansen, 2000; Ponzurick, 1996) e di incentivare la creazione/diffusione di information/knowledge Exchange (Rinallo et al., 2016; Cheng et al., 2014; Li, 2014; De Vanujany et al., 2013; Conway, 2011; 
sinergie Vol. 35, N. 102, 2017

Rinallo e Golfetto, 2011; Ling-Yee, 2007; Lee e Kim, 2008; Ponzurick 1996). Accanto all'importanza delle fiere come strumento del marketing mix, emerge come la letteratura abbia focalizzato la sua attenzione anche su altri due importanti filoni: l'analisi della misurazione delle performance (Alberca-Oliver et al., 2015; Lin et al., 2015; Gottlieb et al., 2014; Tafesse, 2014; Tafesse e Korneliussen, 2012; Rinallo et al., 2010; Ling-Yee, 2007; Hansen, 2004; Tanner, 2002; Munuera et al., 1996) e della organizzazione/ gestione fieristica (Spann et al., 2015; Tafesse e Korneliussen, 2012; Lampel e Meyer, 2008; Horn, 2002; Gregor e Breiter, 2001; Munuera et al., 1996) e lo studio degli stakeholder (Sarmento e Farhangmehr, 2016; Lin et al., 2015; Wong et al., 2014; Gottlieb et al., 2011; Rinallo et al., 2010; Tafesse et al. 2010; Berne e Garcia-Uceda, 2008; Smith et al., 2004; Smith et al., 2003; Rolf Seringhaus e Rosson, 2001; Munuera e Ruiz, 1999).

Suddividendo idealmente i 20 anni di analisi in 3 periodi (1996-2002; 2003-2010; 2011-2016), risulta come alcune aree tematiche, poco o per nulla indagate nel corso della prima fase temporale, abbiano ricevuto una crescente attenzione, da parte della letteratura, durante i due periodi successivi. Nello specifico è infatti a partire dalla seconda fase (2003-2010) e soprattutto nel corso della terza (2011-2016) che la letteratura accademica, dedicata al filone tradeshows/tradefairs, inizia ad approfondire, in maniera sistematica, le seguenti aree tematiche: trade show and opportunities/Risks (Bartosik-Purgat e Schroeder, 2015; Cop e Kara, 2014; Palumbo 2008); trade show and online communication (Hlee et al., 2016; Lapoule e Rowell, 2016; Wu e Wang, 2016; Tafesse, 2014; Kalafsky e Gress, 2013; Conway, 2011; Ling-Yee, 2010, Lee et al., 2008); Trade Show and economic/urban development (Alberca-Oliver et al., 2015; Li, 2014; Kowalik, 2012; Sasaka, 2012; Nunez et al., 2009); trade show and network creation (Measson e Campbell-Hunt, 2015; Sarmento et al., 2015; Li, 2014 Evers e Knight, 2008); trade show and temporary cluster (Rinallo et al., 2016; BartosikPurgat e Schroeder, 2015; Li, 2015; Li, 2014; Rinallo e Golfetto, 2011; Ramirez-Pasillas, 2010); virtual trade show (Remolar et al., 2015; Sharda et al., 2012; Remolar et al., 2011; Geigenmuller, 2010; Mcclure, 2009); trade show and internationalization (Sarmento e Farhangmehr, 2016; Li, 2015; Cheng et al., 2014; Çobanoğlu e Turaeva, 2014; Kalafsky e Gress, 2013; Kreivi et al., 2012; Conway, 2011; Ramirez-Pasillas, 2010; Yuksel e Voola, 2010; Wilkinson et al., 2009; Evers e Knight, 2008; Smith e Smith, 2003); trade show and experential/entertainment component (Wong et al., 2016; Gilliam, 2015; Gottlieb et al., 2014; Andreae et al., 2013; Christopher e Emmanuel, 2012; Rinallo et al., 2010).

Accanto alle aree tematiche caratterizzanti l'intero intervallo di analisi (1996-2016), dalla review emerge, nel corso degli ultimi due archi temporali oggetto di indagine (2002-2010; 2011-2016), un interesse crescente degli studiosi per 1) lo strumento fiera in qualità di piattaforma informativa votata alla creazione di informational/relational network anche e soprattutto in una ottica di crescita/espansione internazionale per le aziende partecipanti (trade show and internationalization); 2) l'importanza delle ricadute dell'evento fiera per le città ospitanti, in termini di trasporti, ristorazione e strutture alberghiere (trade show and economic/urban development); 3) il ruolo crescente assunto dalla comunicazione web e dalla 
virtualizzazione in ambito fieristico (trade show and online communication; virtual trade show) 4) l'importanza attribuita alla componente esperienziale/ di intrattenimento nella organizzazione/gestione dell'evento fieristico (trade show and experential/entertainment component).

In relazione alla prima domanda di ricerca (RQ1), dalla review emergono alcune specifiche tendenze caratterizzanti la letteratura di settore più recente dedicata al filone Trade Shows (TSs). In particolare, accanto al ruolo di primo piano assunto recentemente dallo strumento fiera in qualità di palcoscenico (Andreae et al., 2013) informativo votato alla creazione di relational network (Conway, 2011) in una ottica di opportunità di crescita/ espansione internazionale (De Freitas e Da Silva, 2013; Geigenmuller e Bettis-Outland, 2012) soprattutto per le piccole e medie imprese (Measson e Campbell-Hunt, 2015; Çobanoğlu e Turaeva, 2014), emerge un interesse sempre più evidente, da parte della letteratura recente, verso l'impatto emozionale che l'evento fiera può generare negli stakeholder fieristici (Wong et al., 2016). Lattenzione quindi si sposta dagli aspetti prettamente economici e commerciali dello strumento fiera alla componente emozionale/ esperienziale, in qualità di fattore chiave per la efficacia complessiva delle performance fieristiche (Gilliam, 2015; Gottlieb et al., 2014) e in qualità di uno dei motivi fondamentali di partecipazione, all'evento fiera, da parte dei visitatori (Christopher e Emmanuel, 2012).

Emozionalità questa che deve essere ricercata non solo nella fase di pianificazione/realizzazione dell'evento fisico (Atmosphere, events), ma anche nell'approccio strategico sempre più omnicanale (Lapoule, Rowell, 2016) in grado di gestire i canali offline ed online fieristici (Trade Show websites, Virtual Trade Shows) in maniera sempre più sistematica, garantendo così ai visitatori l'uniformità della esperienza fieristica offline ed online. La rassegna dei contributi internazionali consente inoltre di rispondere alla seconda domanda di ricerca (RQ2), facendo emergere come la comunicazione web assuma oggi un ruolo strategico a sostegno delle manifestazioni fieristiche fisiche (Wu, Wang, 2016), ruolo questo che dipende soprattutto dalla presenza di siti web, in grado di accompagnare gli utenti prima, durante e dopo l'evento fieristico, oltre che dalla qualità dei siti (website structure, content design, user experience). Risulta importante anche la capacità delle fiere virtuali di diventare, a tutti gli effetti, una valida estensione dell'evento fisico (Remolar et al., 2015; Sharda et al., 2012; Remolar et al., 2011; Geigenmuller, 2010), la cui capacità di favorire contatti fisici/reali rappresenta ancora oggi una delle principali caratteristiche di successo dello strumento fiera (Sarmento et al., 2015; Sarmento et al., 2014).

Per approfondire gli aspetti legati alla comunicazione web nel contesto del sistema fieristico italiano di seguito viene descritta l'analisi dei siti web dei quartieri fieristici nazionali. L'indagine è stata realizzata utilizzando il modello 2QCV2Q di Mich e Franch (2000), specificatamente adattato per la valutazione della qualità web fieristica. I dettagli della metodologia sono esposti nel paragrafo successivo.
Tonino Pencarelli

Marco Cioppi

Giancarlo Ferrero

Ilaria Curina

La comunicazione web dei quartieri fieristici 


\section{sinergie}

Vol. 35, N. 102, 2017

\section{Metodologia di ricerca}

Obiettivo dell'analisi è descrivere l'adeguatezza delle azioni di comunicazione $w e b$ adottate dai quartieri fieristici nazionali ${ }^{1}$, attraverso una valutazione della qualità dei loro siti web ufficiali. Il modello 2QCV2Q di Mich e Franch (2000) è lo strumento adottato, con opportuni adattamenti, per la valutazione della qualità dei siti web delle fiere, modello che permette di analizzare qualsiasi sito web indipendentemente dal settore aziendale di studio (Mich e Franch, 2000). Partendo quindi da tale modello e dalla sua struttura generale/dimensioni principali (Identity, Content, Services, Individuation, Management, Usability), si è proceduto con l'inserimento di una serie di quality variables (Tab. 1) specificatamente identificate in letteratura per la valutazione della qualità web fieristica (Lee et al., 2008).

Fig. 2: Modello per la valutazione della qualità dei siti web dei quartieri fieristici

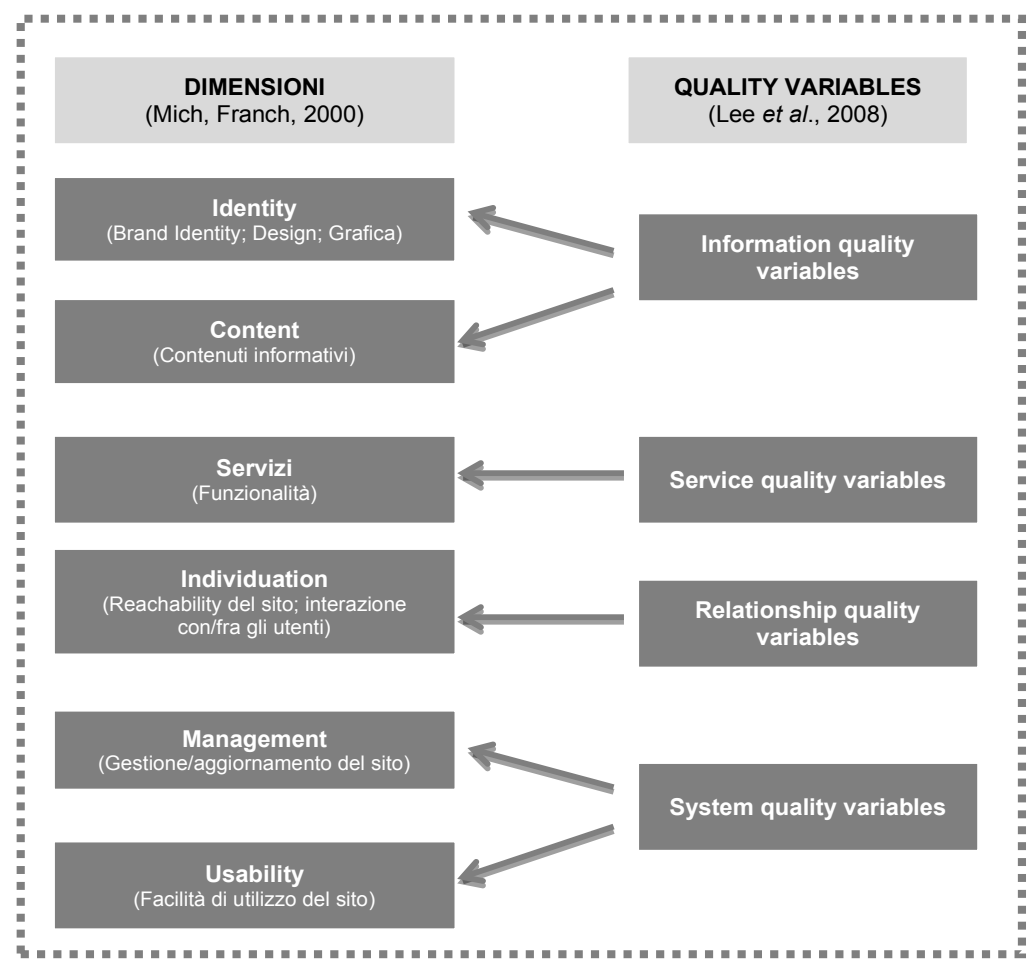

Fonte: Nostra elaborazione

${ }^{1} \quad$ L'Italia conta attualmente 51 quartieri fieristici attivi (Fonte: Nostra elaborazione): Arezzo, Bari, Bassano, Bergamo, Bologna, Bolzano, Brescia, Cagliari, Carrara, Catania, Cesena, Chiuduno, Cremona, Erba, Faenza, Ferrara, Firenze, Foggia, Forlì, Genova, Gonzaga, Lanciano, Lario, Longarone, Lucca, Messina, Milano, Modena, Montichiari, Monza e Brianza, Napoli, Padova, Palermo, Parma, Pesaro, Piacenza, Pordenone, Reggio Emilia, Rimini, Riva del Garda, Roma, Silvi Marina, Sora, Spezia, Torino, Udine e Gorizia, Valenza, Vercelli, Verona, Venezia, Vicenza. 
In seguito alla costruzione del modello, si è proceduto con l'analisi di tutti i siti web dei quartieri fieristici nazionali (49 su 51). Ai fini della valutazione, ad ognuna delle variabili identificate è stato attribuito un punteggio binario in base alla presenza ( 1 punto) o assenza ( 0 punti) delle singole quality variables all'interno dello specifico sito web analizzato.

Tab. 1: Dimensioni e quality variables ${ }^{234}$

\begin{tabular}{|c|c|}
\hline Dimensione & Quality Variables \\
\hline \multirow{3}{*}{ IDENTITY } & $\begin{array}{l}\text { Presenza del logo del quartiere fieristico (Mich, Franch, 2000; Nostra } \\
\text { elaborazione) }\end{array}$ \\
\hline & $\begin{array}{l}\text { Presenza di immagini immediatamente riconducibili al quartiere (Mich, Franch, } \\
\text { 2000; Nostra elaborazione) }\end{array}$ \\
\hline & $\begin{array}{l}\text { Layout del sito coordinato con layout/immagine cartacea (Mich, Franch, 2000; } \\
\text { Nostra elaborazione) }\end{array}$ \\
\hline \multirow{6}{*}{ CONTENT } & Informazioni sul quartiere (Lee et al., 2008; Nostra elaborazione) \\
\hline & Informazioni sui padiglioni (Lee et al., 2008; Nostra elaborazione) \\
\hline & Informazioni sul calendario eventi (Lee et al., 2008; Nostra elaborazione) \\
\hline & Informazioni sui servizi offerti (Lee et al., 2008; Nostra elaborazione) \\
\hline & Informazioni sul territorio/ospitalità (Lee et al., 2008; Nostra elaborazione) \\
\hline & Link esterni (Mich, Franch, 2000) \\
\hline \multirow{5}{*}{ SERVICES } & Form di richiesta (Lee et al., 2008) \\
\hline & Acquisto ticket online (Lee et al., 2008) \\
\hline & Prenotazione online (Lee et al., 2008) \\
\hline & Form di registrazione (Lee et al., 2008) \\
\hline & Area riservata/Privacy (Mich, Franch, 2000) \\
\hline \multirow{7}{*}{ INDIVIDUATION } & Indirizzo del sito facilmente individuabile (Mich, Franch, 2000) \\
\hline & Posizionamento sul motore di ricerca Google (N. pagina) (Mich, Franch, 2000) $)^{2}$ \\
\hline & Presenza dei contatti (Mich, Franch, 2000) \\
\hline & Presenza di community/blog (Mich, Franch, 2000) \\
\hline & Presenza di Newsletter (Mich, Franch, 2000) \\
\hline & Presenza pulsanti social (Mich, Franch, 2000; Nostra elaborazione) \\
\hline & $\begin{array}{l}\text { Presenza di strumenti per la valutazione della customer satisfaction (Mich, } \\
\text { Franch, 2000) }\end{array}$ \\
\hline \multirow{4}{*}{ MANAGEMENT } & Presenza calendario eventi aggiornato $^{3}$ (Mich, Franch, 2000; Nostra elaborazione) \\
\hline & Presenza area stampa aggiornata (Mich, Franch, 2000; Nostra elaborazione) \\
\hline & Presenza news aggiornate (Mich, Franch, 2000; Nostra elaborazione) \\
\hline & Presenza social aggiornati (Mich, Franch, 2000; Nostra elaborazione) \\
\hline \multirow{3}{*}{ USABILITY } & Presenza mappe online del sito (Mich, Franch, 2000) \\
\hline & Rapidità Download Pagine ${ }^{4}$ (Mich, Franch, 2000) \\
\hline & Presenza opzione multilingua (Mich, Franch, 2000) \\
\hline
\end{tabular}

Fonte: Nostra elaborazione

2 Posizionamento nelle prime 3 pagine Google mediante parole chiave "Quartiere fieristico + Regione di appartenenza del polo fieristico": 1 punto; Assenza nelle prime 3 pagine: 0 punti. In generale, gli utenti si fermano alle prime tre pagine di risultati google dopo aver digitato un termine di ricerca (Matthews, 2015).

3 Aggiornamento non superiore a un mese.

$4 \quad$ Valutazione effettuata mediante software di speed test (Web Analyzer). Tempi compresi fra 0,1 e 1 secondo: 1 punto; Tempi superiori a 1 secondo: 0 . Nielsen (2000) valuta i tempi di download compresi fra 0,1 e 1 secondo come lasso di attesa accettabile. 


\section{sinergie}

Vol. 35, N. 102, 2017

\section{Risultati generali}

Nel complesso, su un punteggio massimo raggiungibile pari a 28 punti, solo un quartiere fieristico su 49 ha ottenuto un punteggio superiore a 25 punti, il $48 \%$ dei poli nazionali ha conseguito un punteggio alto (compreso fra $20-25$ punti), mentre il restante $52 \%$ ha raggiunto un punteggio medio (il $44 \%$ fra 10 e 19 punti) e basso (l' $8 \%$ non ha superato i 10 punti complessivi).

Se si tiene conto delle finalità di utilizzo, emergono due distinte tipologie di siti web fieristici: da un lato, siti web prettamente informativi (57\%), che hanno come unico scopo quello di fornire ai propri utenti informazioni dettagliate sulle caratteristiche strutturali del quartiere, sul calendario eventi e sul territorio in cui esso sorge (predominanza della dimensione CONTENT) e, dall'altro, siti web interattivi (43\%) che, accanto a sezioni puramente informative, offrono all'utente unampia gamma di servizi (Es. prenotazione online, acquisto ticket online) e di strumenti relazionali (Es. pulsanti social), il cui obiettivo principale è quello di favorire la creazione di rapporti interattivi e diretti con gli utenti fieristici (predominanza della dimensione SERVICES/INDIVIDUATION).

Per quanto riguarda l'impiego dei Social Network, Facebook si conferma il social più utilizzato dai quartieri fieristici italiani (39 su 49), seguito da Twitter (28), Youtube (19), Google Plus (12), Linkedin (9) e Flickr (5). Agli ultimi posti della classifica social, si attestano Vevo, Foursquare e Vimeo, adottati da un solo quartiere fieristico su 49.

Dai risultati emerge che il $35 \%$ dei poli fieristici offre contenuti dei siti web solo in lingua italiana, mentre il $65 \%$ permette la traduzione dei contenuti in lingua inglese. Solo 4 siti web su 49 sono tradotti anche in tedesco, 3 in francese ed infine solo un quartiere include anche altre lingue (Cinese, Spagnolo, Russo e Arabo).

Dal punto di vista delle singole dimensioni, infine, quelle della IDENTITY, del CONTENT e del MANAGEMENT si confermano gli ambiti maggiormente sviluppati all'interno dei siti web fieristici nazionali, seguite, ad una certa distanza dalla dimensione della INDIVIDUATION. Agli ultimi posti si attestano la dimensione USABILITY e SERVICES (Tab. 2).

Tab. 2: Punteggio singole dimensioni

\begin{tabular}{|l|r|r|r|r|}
\hline Dimensione & $\begin{array}{c}\text { Punteggio } \\
\text { massimo } \\
\text { raggiungibile }\end{array}$ & $\begin{array}{c}\text { Punteggio medio } \\
\text { dell'universo } \\
\text { (Valore assoluto) }\end{array}$ & $\begin{array}{c}\text { Punteggio } \\
\text { medio } \\
\text { dell'universo } \\
(\%)\end{array}$ & $\begin{array}{c}\text { Distanza } \% \\
\text { dal punteggio } \\
\text { massimo }\end{array}$ \\
\hline IDENTITY & 3 & 2,7 & $90 \%$ & $10 \%$ \\
\hline CONTENT & 6 & 4,7 & $78 \%$ & $22 \%$ \\
\hline SERVICES & 5 & 2,1 & $42 \%$ & $58 \%$ \\
\hline INDIVIDUATION & 8 & 4,1 & $51 \%$ & $49 \%$ \\
\hline MANAGEMENT & 4 & 2,9 & $73 \%$ & $27 \%$ \\
\hline USABILITY & 4 & 1,8 & $45 \%$ & $55 \%$ \\
\hline
\end{tabular}

Fonte: Nostra elaborazione 


\section{I siti web distinti per gruppi di strutture fieristiche}

Tonino Pencarelli

Marco Cioppi

Giancarlo Ferrero

Ilaria Curina

Al fine di evidenziare possibili relazioni tra il sistema fieristico

La comunicazione web italiano e le performance di comunicazione online, si è proceduto con la dei quartieri fieristici individuazione/applicazione di una serie di criteri di raggruppamento (UFI, 2014; Commissione Europea, 2013) dei quartieri fieristici nazionali (Tab. 3). In particolare, al fine di individuare specifici cluster, le fiere sono state raggruppate secondo le seguenti variabili: 1) Struttura proprietaria (Natura privata, pubblica o mista); 2) Posizione geografica (Nord, Centro, Sud/Isole); 3) Numero dipendenti $(<10 ; 10-49 ; 50-249$; $>249)$; 4) Fatturato $(<=2 ;<=10$; $<=50 ;>50$ milioni di Euro).

Tab. 3: Criteri di raggruppamento (Dati 2015)

\begin{tabular}{|l|c|c|c|c|}
\hline \multicolumn{5}{|c|}{ Criterio di raggruppamento } \\
\hline Posizione geografica & Nord & Centro & Sud & \\
& $69 \%$ & $16 \%$ & $15 \%$ & \\
\hline Struttura proprietaria & Capitale & Capitale & Capitale privato & \\
& pubblico & misto & $15 \%$ & \\
& $46 \%$ & $39 \%$ & & \\
\hline Dimensione & Micro & Piccola & Media & Grande \\
& $35 \%$ & $44 \%$ & $15 \%$ & $6 \%$ \\
\hline Fatturato & $<=2$ & $<=10$ & $<=50$ & $>50$ \\
& $43 \%$ & $33 \%$ & $15 \%$ & $9 \%$ \\
\hline
\end{tabular}

Fonte: Nostra elaborazione (Dati AIDA/Contatto diretto)

Sotto il profilo della struttura proprietaria, risulta che il $46 \%$ dei quartieri fieristici nazionali è di natura pubblica, contro il 39\% a capitale misto (pubblico e privato). Solo il 15\% dei poli presenta una struttura di natura esclusivamente privata. A livello geografico, la maggior parte dei quartieri nazionali (69\%) sorge nel Nord Italia, a scapito delle zone centrali (16\%) e meridionali/insulari della Penisola (15\%). In una ottica dimensionale, invece, l'analisi mette in luce una netta prevalenza di quartieri fieristici di piccole (44\%) e di micro dimensioni (35\%), seguiti ad una certa distanza da strutture di medie (15\%) e di grandi dimensioni (6\%). Dal punto di vista del fatturato, infine, se il 76\% dei poli ha conseguito nel 2015 un fatturato non superiore ai 10 milioni di Euro, solo una ridotta percentuale dell'universo fieristico (9\%) ha raggiunto un fatturato superiore ai 50 milioni di Euro.

La Tabella 4 mostra i punteggi medi perseguiti dai diversi gruppi fieristici identificati (Struttura proprietaria, Posizione geografica, Dipendenti, Fatturato) rispetto al punteggio massimo perseguibile (28 punti). 
sinergie Vol. 35, N. 102, 2017

Tab. 4: Gruppi fieristici e punteggio medio complessivo

\begin{tabular}{|c|c|}
\hline Forma Giuridica & Punteggio Medio \\
\hline Pubblica & $18 / 28$ \\
\hline Privata & $24 / 28$ \\
\hline Mista & $21 / 28$ \\
\hline Posizione geografica & Punteggio Medio \\
\hline Nord & $20,1 / 28$ \\
\hline Centro & $19,6 / 28$ \\
\hline Sud/Isole & $17,3 / 28$ \\
\hline Dimensione & Punteggio Medio \\
\hline Micro & $16,5 / 28$ \\
\hline Piccola & $20,8 / 28$ \\
\hline Media & $22,5 / 28$ \\
\hline Grande & $23 / 28$ \\
\hline Fatturato & Punteggio Medio \\
\hline$<=2$ Milioni di Euro & $15,9 / 28$ \\
\hline$<=10$ Milioni di Euro & $21,4 / 28$ \\
\hline$<=50$ Milioni di Euro & 23 \\
\hline$>50$ Milioni di Euro & 23,6 \\
\hline
\end{tabular}

Fonte: Nostra elaborazione

Nel complesso, i punteggi complessivi più elevati sono stati raggiunti dai quartieri fieristici:

- di natura privata;

- collocati nel Nord della penisola;

- di grande dimensione (Oltre 250 addetti);

- con un fatturato superiore ai 50 milioni di Euro.

A livello di singole dimensioni, emerge come i quartieri con struttura proprietaria privata abbiano conseguito il punteggio più elevato (rispetto ai poli con capitale pubblico e misto) grazie soprattutto agli ambiti del CONTENT, SERVICES e MANAGEMENT. CONTENT e USABILITY rappresentano, invece, le dimensioni che hanno permesso ai quartieri del Nord Italia di posizionarsi al primo posto, mentre per quanto riguarda i quartieri di grandi dimensioni (oltre 250 addetti ed un fatturato superiore ai 50 Milioni di Euro), gli ambiti del CONTENT, MANAGEMENT e USABILITY rappresentano le dimensioni che, più di altre, hanno permesso a tali gruppi fieristici di superare i poli di medie, piccole e di micro dimensioni.

\section{Discussione dei risultati}

Nel complesso, la quasi totalità dei quartieri fieristici nazionali (96\%) possiede un sito web, risultato questo che mette in evidenza come il comparto sia comunque consapevole dell'importanza di essere oggi presenti online.

$\mathrm{Da}$ unanalisi più dettagliata emerge come esista una netta differenziazione fra i quartieri che hanno ottenuto un punteggio alto e 
quelli che, al contrario, hanno conseguito un punteggio medio-basso. In particolare, se da un lato il $48 \%$ ha ottenuto un punteggio elevato (compreso fra i 20 ed i 25 punti), la restante percentuale $(52 \%)$ ha conseguito un punteggio medio (il $44 \%$ ha ottenuto un punteggio compreso fra 10 e 19) e basso (l' $8 \%$ non ha superato i 10 punti). Solo un quartiere fieristico, sul totale analizzato, ha ottenuto un punteggio superiore a 25 .

Dal punto di vista delle singole dimensioni indagate, i siti web presentano una forte Brand identity (IDENTITY), con la presenza in Home Page del logo/immagini immediatamente riconducibili al quartiere fieristico, unelevata ricchezza contenutistica - CONTENT (offerta di informazioni sul quartiere, sul calendario eventi e sul territorio in cui sorge il polo) e (anche se in misura minore) una corretta ed attenta gestione/aggiornamento delle informazioni presenti al loro interno (MANAGEMENT).

Al contrario, le dimensioni della INDIVIDUATION, della USABILITY e dei SERVICES risultano gli ambiti meno performanti dei siti web osservati (Fig. 3). In sostanza, i quartieri nazionali sembrano essere meno inclini a 1) investire in SEO; 2 ) incrementare la costruzione di rapporti diretti ed interattivi con/fra gli utenti (mediante l'offerta di chat, blog o una stretta connessione sito internet e social); 3) migliorare la navigabilità del sito (attraverso, ad esempio, l'inclusione di mappe online o attraverso la possibilità di leggere i contenuti in più lingue); 4) arricchire il sito di funzionalità online (Es. acquisto online ticket, prenotazioni online).

Fig. 3: Punteggio singole dimensioni

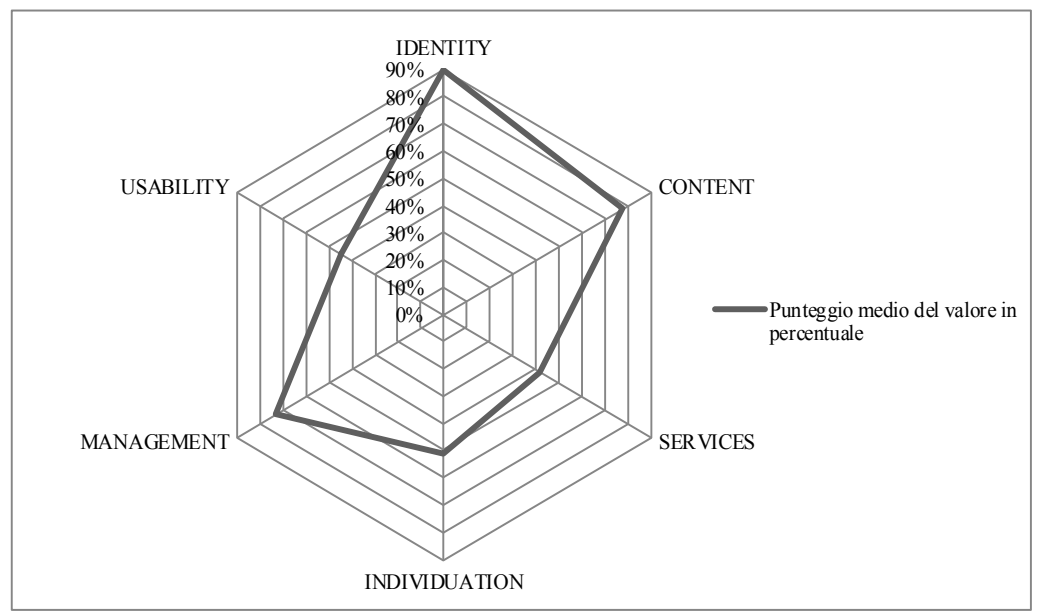

Fonte: Nostra elaborazione

Nonostante i punteggi medi più elevati siano stati conseguiti dai quartieri nazionali più grandi (oltre 250 addetti/fatturato superiore ai 50 milioni di Euro), emerge come, nelle prime due posizioni della classifica generale, compaiano quartieri fieristici di micro/piccole dimensioni e con un fatturato inferiore ai 50 milioni di Euro (Tab. 5).
Tonino Pencarelli Marco Cioppi

Giancarlo Ferrero

La comunicazione web dei quartieri fieristici 
Sinergie

Vol. 35, N. 102, 2017
Tab. 5: Classifica generale (Prime tre posizioni)

\begin{tabular}{|c|c|c|c|c|c|}
\hline Podio & Capitale & $\begin{array}{c}\text { Posizione } \\
\text { geografica }\end{array}$ & $\begin{array}{c}\text { Numero } \\
\text { Addetti }\end{array}$ & Fatturato & Pt. Tot. \\
\hline 1 & Privato & Nord & P & $<=50$ & $26 / 28$ \\
\hline 2 & Privato & Centro & P & $>=10$ & $25 / 28$ \\
\hline 2 & Misto & Nord & G & $>50$ & $25 / 28$ \\
\hline 2 & Misto & Nord & Micro & $<=10$ & $25 / 28$ \\
\hline 2 & Misto & Nord & M & $>50$ & $25 / 28$ \\
\hline 2 & Misto & Nord & P & $<=10$ & $25 / 28$ \\
\hline 3 & Misto & Nord & M & $<=50$ & $24 / 28$ \\
\hline
\end{tabular}

Fonte: Nostra elaborazione

Dai risultati emerge dunque un interrogativo: è possibile anche per una piccola struttura fieristica realizzare e gestire siti web di qualità? Tali quartieri fieristici rappresentano infatti imprese best in class che testimoniano come, non solo i poli di grandi dimensioni, ma anche aziende di piccole/micro dimensioni possano essere in grado di adottare strategie comunicative online ben progettate e pianificate.

Dall'analisi della classifica generale emerge come la maggioranza delle piccole strutture non sembra in grado di sfruttare appieno le potenzialità offerte dalla rete, limitandosi ad offrire siti web vetrina. Nel complesso, molteplici possono essere le cause che conducono le imprese fieristiche di piccole dimensioni verso questa quasi generalizzata situazione di sottoutilizzo delle potenzialità del web ed in particolare:

- scarsa numerosità di risorse umane;

- mancanza di competenze specializzate all'interno del personale;

- mancanza di risorse economiche da dedicare;

- percezione di dover affrontare investimenti particolarmente elevati e, di conseguenza, non alla loro portata.

\section{Conclusioni e implicazioni teoriche e manageriali della ricerca}

La ricerca ha permesso di rispondere ai tre quesiti iniziali da cui è partito lo studio. Per quanto riguarda il primo (Quali sono le principali tendenze emergenti dalla recente letteratura di settore dedicata al filone Trade Shows?), dalla review emerge come Internationalization, Network building, Entertainment component e Online communication \& Virtualization rappresentino le principali tendenze caratterizzanti la letteratura di settore più recente (2011-2016) dedicata ai Trade Shows.

Relativamente al secondo quesito della ricerca (Alla luce delle tendenze in atto, che ruolo assume la comunicazione web all'interno dell'attuale scenario fieristico internazionale?), lo studio ha evidenziato come la comunicazione web stia assumendo sempre più, in ambito fieristico, una importanza strategica a livello internazionale, in qualità di strumento di supporto e di completamento della esperienza fieristica fisica.

Per quanto riguarda, infine, la terza domanda di ricerca (Alla luce del ruolo assunto dalla comunicazione web in ambito fieristico, $i$ poli fieristici 
nazionali adottano attività di comunicazione web adeguate?), dalla analisi dei siti web degli exhibition centers italiani emerge nel complesso un impiego ancora deficitario ed arretrato della comunicazione online: pochi infatti (meno della metà dell'universo indagato) sono i quartieri che adottano attività comunicative online realmente interattive, limitandosi, nella maggior parte dei casi, ad offrire siti web vetrina, aventi come unico scopo quello di comunicare agli utenti la loro esistenza in Rete.

Dal lavoro emergono alcune implicazioni manageriali. Lo studio individua una serie di possibili percorsi di apprendimento e di innovazione che il comparto fieristico nazionale potrebbe intraprendere per ridurre il gap che attualmente lo separa da un uso totalmente performante degli strumenti comunicativi del web.

In particolare, diventa fondamentale, per i quartieri fieristici nazionali, comprendere come sia assolutamente imprescindibile l'adozione di politiche comunicative realmente interattive a due vie (mediante quindi la creazione e gestione di siti web che consentano un reale dialogo fra gli utenti) e come, di conseguenza, sia necessario superare la concezione "sito vetrina = esistenza online per l'utente", in quanto oggi possedere un sito statico/vetrina non sarà più sufficiente a richiamare nuovi interlocutori fieristici e/o a fidelizzare quelli già esistenti.

Il modello di valutazione di qualità esposto rappresenta inoltre uno strumento di management operativo utile per identificare le aree di criticità/ miglioramento dei siti web fieristici.

In particolare, dall'analisi empirica emerge come le dimensioni dei SERVICES, INDIVIDUATION, del MANAGEMENT e della USABILITY rappresentino le aree maggiormente critiche dei siti web dei quartieri fieristici italiani, per le quali si possono mettere in luce significative aree di miglioramento (Tab. 6).

Tab. 6: Aree di miglioramento: Possibili interventi

\begin{tabular}{|l|l|}
\hline Aree di miglioramento & \multicolumn{1}{c|}{ Possibili interventi } \\
\hline SERVICES & $\begin{array}{l}\text { Maggior orientamento commerciale (inclusione all'interno del sito web } \\
\text { di funzionalità online come la possibilità di acquistare i biglietti online, } \\
\text { prenotare una accomodation per il periodo di permanenza in fiera, ecc.). }\end{array}$ \\
\hline INDIVIDUATION & $\begin{array}{l}\text { Maggior orientamento interattivo mediante l'investimento in risorse } \\
\text { umane specificatamente dedicate alle strategie di Social Media Marketing } \\
\text { (inclusione/gestione di funzionalità che consentono di incentivare la } \\
\text { creazione di rapporti interattivi e diretti con/fra gli utenti). }\end{array}$ \\
\hline MANAGEMENT & $\begin{array}{l}\text { Necessità di investire in risorse umane (interne/outsourcing) per } \\
\text { garantire una gestione continua ed un aggiornamento costante dei } \\
\text { contenuti/struttura del sito web. }\end{array}$ \\
\hline USABILITY & $\begin{array}{l}\text { Necessità di aprirsi a livello internazionale (offrire, ad esempio, all'utente } \\
\text { la possibilità di leggere i contenuti del sito in più lingue); Superamento } \\
\text { della filosofia "Think local, act local". }\end{array}$ \\
\hline
\end{tabular}

Fonte: Nostra elaborazione

In particolare, i responsabili delle strutture fieristiche italiane sono chiamati a:

- accrescere la consapevolezza circa l'importanza della comunicazione web in qualità distrumento di supporto/promozione e di commercializzazione fieristica; 
sinergie Vol. 35, N. 102, 2017

avviare processi di innovazione volti ad investire maggiormente nella realizzazione/gestione di siti web interattivi, canali potenzialmente in grado, molto più di altri, di avvicinare nuovi interlocutori fieristici e/o fidelizzare quelli già esistenti;

- sviluppare, accanto alle competenze professionali tradizionali (allestimento, logistica, accoglienza, ecc.), nuove e diversificate capacità di natura comunicativa e relazionale mediante l'inserimento di figure specializzate nella comunicazione online/social.

Per avviare azioni di comunicazione online performanti si rendono necessarie molteplici nuove competenze specializzate: 1) strategiche (pianificazione della strategia; integrazione sito web/altri strumenti di comunicazione adottati); 2) di gestione/management (content management; competenze per svolgere attività di copy e azioni di aggiornamento dei canali comunicativi online adottati). Non potendo, inoltre, essere sempre gestite all'interno delle strutture fieristiche (soprattutto nel caso dei quartieri di piccole dimensioni), queste competenze potranno essere incluse mediante processi di outsourcing/sviluppo di networking.

In definitiva, diventa fondamentale, per i quartieri nazionali, comprendere come la promozione delle proprie strutture e delle singole manifestazioni in calendario debba essere sempre accompagnata da una comunicazione web costante (allo scopo di seguire i propri interlocutori prima, durante e dopo la chiusura degli eventi fieristici) e dalla consapevolezza che un aggiornamento saltuario e sporadico del proprio sito web/profili social non sarà sufficiente a richiamare nuovi interlocutori e/o fidelizzare quelli già esistenti.

Il principale limite del lavoro si collega al fatto che l'indagine è stata realizzata da una prospettiva esterna (mediante la valutazione dei siti web aziendali dei poli fieristici nazionali), non rendendo di conseguenza possibile, in questo ambito, effettuare una valutazione della comunicazione web in relazione alle risorse e agli specifici obiettivi delle governance fieristiche (valutazione necessaria per capire se le azioni comunicative adottate dai poli indagati siano consapevoli o meno).

Per il futuro, è interesse degli autori estendere la ricerca sia da un punto di vista metodologico (mediante interviste dirette ai responsabili dei poli fieristici nazionali), che geografico (mediante un confronto con i sistemi fieristici di altri paesi, allo scopo di capire l'effettivo stato di sviluppo della comunicazione web fieristica nazionale).

\section{Bibliografia}

AEFI (2014-2016), Osservatorio congiunturale sul Settore Fieristico.

AHOLA E.K. (2012), "Towards an understanding of the role of Trade Fairs as facilitators of consumer creativity", Journal of Marketing Communications, vol. 18 , n. 5, pp. 321-333.

ALBERCA-OLIVER P., RODRÍGUEZ-OROMENDÍA A., PARTE-ESTEBAN L. (2015), "Measuring the efficiency of Trade Shows: A Spanish case study", Tourism Management, vol. 47, pp. 127-137. 
ALOUI C. (2016), "Optimal capacity sharing of a two two sided monopoly platform: the case of a Trade Fair", Economic Modelling, vol. 54, pp. 276-288.

ANDREAE M., HSU J.Y., NORCLIFFE G. (2013), "Performing the Trade Show: The case of the Taipei International Cycle Show", Geoforum, vol. 49, pp. 193-201. dei quartieri fieristici

BARTOSIK-PURGAT M., SCHROEDER J. (2015), "International Trade Fairs as a source of information on foreign markets and partners", International Business and Global Economy, n. 33, pp. 519-529.

BERNE C., GARCIA-UCEDA M.E. (2008), "Criteria involved in evaluation of Trade Shows to visit", Industrial Marketing Management, vol. 37, n. 5, pp. 565-579.

BLACK R. (1986), “The Trade Show Industry: Management and Marketing Career Opportunities", Trade Show Bureau, East Orleans, MA.

BLYTHE J. (2009), “Trade Fairs as communication: a new model”, Journal of Business and Industrial Marketing, vol. 25, n. 1, pp. 57-62.

BLYTHE J. (2002), "Using Trade Fairs in key account management", Industrial Marketing Management, vol. 31, n. 7, pp. 627-635.

CHENG H., KOIVISTO E., MATTILA P. (2014), "International fashion Trade Shows as knowledge creation platforms for microenterprises", Journal of Global Fashion Marketing, vol. 5, n. 2, pp. 149-164.

CHRISTOPHER M., EMMANUEL L. (2012), "Visitors' objectives for attending a regional Trade Fair in Ghana", European Journal of Social Sciences, vol. 31, n. 4, pp. 496-506.

CHU M.C., CHIU S.M. (2013), "Effective marketing strategies to attract business visitors at Trade Shows", International Journal of Business and Management, vol. 8, n. 24, pp. 64-72.

ÇOBANOGLU E., TURAEVA V. (2014), "Effects of the Pre-show, At-show and Postshow Firm Activities on Trade Show Performance Measurement”, ProcediaSocial and Behavioral Sciences, vol. 150, pp. 762-771.

COMMISSIONE EUROPEA (2013), Definizione di PMI, disponibile al seguente indirizzo:http://ec.europa.eu/competition/consultations/2013_ consolidated_gber/annex_II_it.pdf.

CONWAY C. (2011), "The role of international Trade Shows in supporting small firm internationalization", The International Journal of Entrepreneurship and Innovation, vol. 12, n. 3, pp. 213-213.

COP R., KARA R.T. (2014), "The role of Trade Fairs in industrial marketing: a research on defence industry Trade Fairs", Journal of Management Marketing and Logistics, vol. 1, n. 3, pp. 156-172.

DE FREITAS SANTOS J., DA SILVA P.B.M. (2013), "Participate (or not) in international Trade Fairs? Decision factors of Portuguese managers", Revista de Management Comparat International, vol. 14, n. 5, pp. 689-703.

DE VAUJANY F.X., CARTON S., DOMINGUEZ-PERY C., VAAST E. (2013). "Moving closer to the fabric of organizing visions: The case of a Trade Show", The Journal of Strategic Information Systems, vol. 22, n. 1, pp. 1-25.

EUROPEAN COMMISSION, (2015), "Annual report on European SMEs", disponibile al seguente indirizzo: http://ec.europa.eu/growth/smes/ business-friendly-environment/performance-review_en.

EVERS N., KNIGHT J. (2008), "Role of international Trade Shows in small firm internationalization: a network perspective", International Marketing Review, vol. 25 , n. 5, pp. 544-562. 
GEINGENMULLER A., BETTIS-OUTLAND H. (2012), "Brand equity in B2B services and consequences for the Trade Show industry", Journal of Business and Industrial Marketing, vol. 27, n. 6, pp. 428-435.

GEIGENMULLER A. (2010), "The role of virtual Trade Fairs in relationship value creation", Journal of Business and Industrial Marketing, vol. 25, n. 4, pp. 284292.

GILLIAM D.A. (2015), “Trade Show boothscapes”, Journal of Marketing Management, vol. 31, n. 17/18, pp. 1878-1898.

GOPALAKRISHNA S., ROSTER C.A., SRIDHAR S. (2010), "An exploratory study of attendee activities at a business Trade Show", Journal of Business Industrial Marketing, vol. 25, n. 4, pp. 241-248.

GOTTLIEB U., BROWN M., FERRIER L. (2014), "Consumer perceptions of Trade Show effectiveness: Scale development and validation within a B2C context", European Journal of Marketing, vol. 48, n. 1/2, pp. 89-107.

GOTTLIEB U.R., BROWN M.R., DRENNAN J. (2011), "The influence of service quality and Trade Show effectiveness on post-show purchase intention", European Journal of Marketing, vol. 45, n. 11/12, pp. 1642-1659.

HANSEN K. (2004), "Measuring performance at Trade Shows: scale development and validation", Journal of business Research, vol. 57, n. 1, pp. 1-13.

HANSEN K. (2000), "From selling to relationship marketing at international Trade Fairs", Journal of Convention and Exhibition Management, vol. 2, n. 1, pp. 37-53.

HERBIG P., O’HARA B., PALUMBO F.A., (1998), “Trade Show: who, what, why”, Marketing Intelligence and Planning, vol. 16, n. 7, pp. 425-435.

HERBIG P., O’HARA B., PALUMBO F. (1997), “Differences between Trade Show exhibitors and non-exhibitors", Journal of Business and Industrial Marketing, vol. 12, n. 6, pp. 368-382.

HLEE S., LEE J., MOON D., YOO C. (2016), “The acceptance of 'intelligent Trade Shows': Visitors' evaluations of IS innovation”, Information Systems Frontiers, doi:10.1007/s10796-016-9703-5, pp. 1-13.

HORN L. (2002), "Making Trade Shows pay off: Utilizing promotional gifts rather than giveaways", Journal of Promotion Management, vol. 8, n. 1, pp. 127-136.

KALAFSKY R.V., GRESS D.R. (2013), "Trade Fairs as an export marketing and research strategy: Results from a study of Korean advanced machinery firms", Geographical Research, vol. 51, n. 3, pp. 304-317.

KIRCHGEORG M., JUNG K., KLANTE O. (2010), “The future of Trade Shows: insights from a scenario analysis", Journal of Business and Industrial Marketing, vol. 25, n. 4, pp. 301-312.

KIRCHGEORG M., SPRINGER C., KASTNER, E. (2009), "Objectives for successfully participating in Trade Shows", Journal of Business and Industrial Marketing, vol. 25, n. 1, pp. 63-72.

KOWALIK I. (2012), "Influence of Trade Fairs on a Host City Brand", Public Policy and Administration, vol. 11, n. 4, pp. 629-640.

KREIVI M., WANG L., MUHOS M., KESS P. (2012), "Trade Fairs and NetworkingSMES Internationalizing in China”, Journal of US-China Public Administration, vol. 9, n. 5, pp. 516-533.

LAMPEL J., MEYER A.D. (2008), “Guest editors' introduction: Field-configuring events as structuring mechanisms: How conferences, ceremonies, and Trade Shows constitute new technologies, industries, and markets", Journal of Management Studies, vol. 45, n. 6, pp. 1025-1035. 
LAPOULE P., ROWELL J. (2016), “Using Social Media to Support Trade Shows: Developing the Capabilities", South Asian Journal of Business and Management Cases, vol. 5, n. 1, pp. 88-98, In Press.

LEE C.H., KIM S.Y. (2008), "Differential effects of determinants on multi-dimensions Tonino Pencarell Marco Cioppi Giancarlo Ferrero Ilaria Curina La comunicazione web dei quartieri fieristici of Trade Show performance: By three stages of pre-show, at-show, and postshow activities", Industrial Marketing Management, vol. 37, n. 7, pp. 784-796.

LEE J., LOVE C., HAN T. (2008), “Trade Show Websites: An Examination of Critical Websites' Quality Factors and Content Items", Journal of Convention and Event Tourism, vol. 9, n. 1, pp. 35-59.

LI Y. (2015), "Trade Shows in the Globalizing Knowledge Economy", Journal of Economic Geography, vol. 15, n. 4, pp. 841-842.

LI P.F. (2014), "Global temporary networks of clusters: structures and dynamics of Trade Fairs in Asian economies", Journal of Economic Geography, doi: 10.1093/jeg/lbu009.

LIN Y., JIANG J., KERSTETTER D. (2015), "A Three-Component Framework for Trade Show Performance Evaluation”, Journal of Hospitality and Tourism Research, 1096348015619747.

LING-YEE L. (2010), "Antecedents and effect of internet implementation for Trade Shows", Journal of Business and Industrial Marketing, vol. 25, n. 4, pp. 272-283.

LING-YEE L. (2007), "Marketing resources and performance of exhibitor firms in Trade Shows: A contingent resource perspective", Industrial Marketing Management, vol. 36, n. 3, pp. 360-370.

LING-YEE L. (2006), "Relationship learning at Trade Shows: Its antecedents and consequences", Industrial Marketing Management, vol. 35, n. 2, pp. 166-177.

MATTHEWS J. (2015), Posizionarsi in prima pagina su Google - Consigli SEO per il Marketing online, BabeIcube Inc., London.

MCCLURE M. (2009), "Virtual Trade Shows: a realistic alternative to business travel?", EContent, vol. 32, n. 1, pp. 34-38.

MEASSON N., CAMPBELL-HUNT C. (2015), "How SMEs use Trade Shows to enter global value chains", Journal of Small Business and Enterprise Development, vol. 22, n. 1, pp. 99-126.

MENON S., MANOJ E. (2013), "A Study of Exhibitor Firms at a Tourism Trade Show", International Journal of Marketing and Business Communication, vol. 2, n. 2, pp. 64-73.

MICH L., FRANCH M. (2000), "2QCV2Q: a model for web sites design and evaluation", In IRMA Conference, pp. 586-589.

MUNUERA J.L., RUIZ S. (1999), “Trade Fairs as services: a look at visitors' objectives in Spain”, Journal of Business Research, vol. 44, n. 1, pp. 17-24.

MUNUERA J.L., HERNANDEZ M., MAYA S.R. (1996), "Planning of Trade Shows as Marketing Activities", International Journal of Research in Marketing, vol. 3, n. 13, pp. 298.

NIELSEN J. (2000), Web Usability, Apoegeo Editore, Milano.

NÚÑEZ M., GARCÍA-LOZANO R., BOQUERA P., GABARRELL X., RIERADEVALL J. (2009); "Temporary structures as a generator of waste in covered Trade Fairs", Waste management, vol. 29, n. 7, pp. 2011-2017.

PALUMBO F.A. (2008), “Trade Show/Fair piracy and industrial espionage”, Journal of Convention and Event Tourism, vol. 9, n. 4, pp. 277-292.

PALUMBO F., HERBIG P.A. (2002), "Trade Shows and Fairs: An important part of the international promotion mix", Journal of Promotion Management, vol. 8, n. 1, pp. 93-108. 
PONZURICK T.G. (1996), “International buyers perspective toward Trade Shows and other promotional methods", Journal of Marketing Theory and Practice, vol. 4, n. 1, pp. 9-19.

POWER D., JANSSON J. (2008), "Cyclical clusters in global circuits: Overlapping spaces in furniture Trade Fairs”, Economic Geography, vol. 84, n. 4, pp. 423448.

PRADO-ROMÁN C., BLANCO-GONZÁLEZ A., DÍEZ-MARTÍN F. (2012), "Efficiency of the exhibitors at art Trade Show", International Journal of Arts and Commerce, vol. 1, n. 6, pp. 47-54.

RAMÍREZ-PASILLAS M. (2010), "International Trade Fairs as amplifiers of permanent and temporary proximities in clusters", Entrepreneurship and Regional development, vol. 22, n. 2, pp. 155-187.

REMOLAR I., GARCÉS A., REBOLLO C., CHOVER M., QUIRÓS R., GUMBAU J. (2015), "Developing a virtual Trade Fair using an agent-oriented approach", Multimedia Tools and Applications, vol. 74, n. 13, pp. 4561-4582.

REMOLAR I., CHOVER M., QUIRÓS R., GUMBAU J., CASTELLÓ P., REBOLLO C., RAMOS F. (2011), "Design of a multiuser virtual Trade Fair using a game engine”, Transactions on Computational Science, vol. 6670, Special Issue, pp. 118-139.

RINALLO D., BATHELT H., GOLFETTO F. (2016), "Economic geography and industrial marketing views on Trade Shows: Collective marketing and knowledge circulation", Industrial Marketing Management, In Press.

RINALLO D., GOLFETTO F. (2011), "Exploring the knowledge strategies of temporary cluster organizers: A longitudinal study of the EU fabric industry Trade Shows (1986-2006)", Economic Geography, vol. 87, n. 4, pp. 453-476.

RINALLO D., BORGHINI S., GOLFETTO F. (2010), "Exploring visitor experiences at Trade Shows", Journal of Business and Industrial Marketing, vol. 25, n. 4, pp. 249-258.

RODRIGUEZ OROMENDÍA A., PAZ R., DOLORES M., RUFÍN R. (2015), "Research note: Relationship versus transactional marketing in travel and tourism Trade Shows", Tourism Economics, vol. 21, n. 2, pp. 427-434.

ROLF SERINGHAUS F.R., ROSSON P.J. (2001), "Firm experience and international Trade Fairs”, Journal of Marketing Management, vol. 17, n. 7/8, pp. 877-901.

SARMENTO M., FARHANGMEHR M. (2016), "Grounds of Visitors Post Trade Fair Behavior: An Exploratory Study", Journal of Promotion Management, pp. 1-16.

SARMENTO M., SIMÕES C., FARHANGMEHR M. (2015), "Applying a relationship marketing perspective to B2B Trade Fairs: The role of socialization episodes", Industrial Marketing Management, vol. 44, (2015), pp. 131-141.

SARMENTO M., SIMES C., FARHANGMEHR M. (2014), "B2B Interactions at Trade Fairs and Relationship Quality: A Conceptual Approach. Field Guide to Case Study Research in Business-to-Business Marketing and Purchasing", Advances in Business Marketing and Purchasing, vol. 21, Emerald Group Publishing Limited, pp. 167-189.

SASAKA P.S. (2012), "The Effectiveness of Trade Shows and Exhibitions as Organizational Marketing Tool (Analysis of Selected Companies in Mombasa)", International Journal of Business and Social Science, vol. 3, n. 22, pp. 219-230. 
SHARDA R., SIKOLIA D., THOMAS J., SAMBASIVAN R. (2012), "A Design Science Approach to Virtual World Implementation of Trade Fairs", Pacific Asia Journal of the Association for Information Systems, vol. 4, n. 2, pp. 49-70.

SMITH T.M., GOPALAKRISHNA S., SMITH P.M. (2004), "The complementary Tonino Pencarelli Marco Cioppi Giancarlo Ferrero Ilaria Curina La comunicazione web dei quartieri fieristici effect of Trade Shows on personal selling", International Journal of Research in Marketing, vol. 21, n. 1, pp. 61-76.

SMITH T.M., HAMA K., SMITH P.M. (2003), "The effect of successful Trade Show attendance on future show interest: exploring Japanese attendee perspectives of domestic and offshore international events", Journal of Business and Industrial Marketing, vol. 18, n. 4/5, pp. 403-418.

SØILEN K.S. (2010), "Boosting innovation and knowledge through delocalization: market intelligence at Trade Shows", Problems and Perspectives in Management, vol. 8, n. 3, pp. 200-207.

SPANN M.S., JOHNSON L., ADAMS M., BENDALL D. (2015), "Trade Show Planning: A Model and Tools for Maximizing Effectiveness", Journal of Small Business Strategy, vol. 4, n. 2, pp. 17-30.

TAFESSE W., SKALLERUD K. (2015), "Towards an exchange view of Trade Fairs", Journal of Business and Industrial Marketing, vol. 30, n. 7, pp. 795-804.

TAFESSE W. (2014), "Understanding how resource deployment strategies influence Trade Show organizers' performance effectiveness", European Journal of Marketing, vol. 48, n. 5/6, pp. 1009-1025.

TAFESSE W., KORNELIUSSEN T. (2012), "Identifying factors affecting consumers purchase incidence at retail Trade Shows", Journal of Retailing and Consumer Services, vol. 19, n. 4, pp. 438-444.

TAFESSE W., KORNELIUSSEN T. (2011), "The dimensionality of Trade Show performance in an emerging market", International Journal of Emerging Markets, vol. 6, n. 1, pp. 38-49.

TAFESSE W., KORNELIUSSEN T., SKALLERUD K. (2010), "Importance performance analysis as a Trade Show performance evaluation and benchmarking tool", Journal of Convention and Event Tourism, vol. 11, n. 4, pp. 314-328.

TANNER J.F. (2002), "Leveling the playing field: factors influencing Trade Show success for small companies", Industrial Marketing Management, vol. 31, n. 3, pp. 229-239.

UFI (2014-2016), Global Exhibition Industry Statistics.

WILKINSON T.J., BROUTHERS L.E., SALAZAR D., MCNALLY M. (2009), "The strategic impact of international Trade Shows and trade missions for entrepreneurial firms", Journal for Global Business Advancement, vol. 2, n. 3, pp. 207-220.

WONG J., LI T., CHEN A., PENG N.S. (2016), "The effects of Trade Show environments on visitors", Event Management, In Press.

WONG J.Y., LI T.H., PENG N., CHEN A.H. (2014), "Conceptualizing Trade Show visitors' consumption behavior", International Journal of Tourism Research, vol. 16 , n. 4 , pp. 325-328.

WU X., WANG C. (2016), "Research on designing the official websites of Trade Shows based on user experience", Journal of Convention and Event Tourism, vol. 17, n. 3, pp. 234-246.

YUKSEL U., VOOLA R. (2010), “Travel Trade Shows: exploratory study of exhibitors' perceptions", Journal of Business and Industrial Marketing, vol. 25, n. 4, pp. 293-300. 


\section{sinergie}

Vol. 35, N. 102, 2017

Academic or professional position and contacts

Tonino Pencarelli

Full Professor of Management

University of Urbino Carlo Bo - Italy

e-mail: tonino.pencarelli@uniurb.it

Marco Cioppi

Associate Professor of Management

University of Urbino Carlo Bo - Italy

e-mail: marco.cioppi@uniurb.it

Giancarlo Ferrero

Full Professor of Management

University of Urbino Carlo Bo - Italy

e-mail: giancarlo.ferrero@uniurb.it

Ilaria Curina

PhD Student of Management

University of Urbino Carlo Bo - Italy

e-mail: ilaria.curina@uniurb.it

sinergie

italian journal of management

ISSN 0393-5108 DOI 10.7433/s102.2017.14 pp. $209-230$

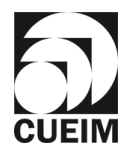

